large; it was situated immediately between the crura of the diaphragm, between which it passed upwards until it came into contact with the inferior lobe of the lung; the aperture in the artery was in its anterior wall. There was no perforation of the diaphragm by absorption, but the tumor had forced its way upwards between the crura, merely dissecting through cellular connections.

Dr. Stokes concluded by remarking, that there were three circumstances in this case which rendered it of great value; first, the diagnosis of the aneurysm from symptoms; second, the confirmation of the stethoscopic signs; third, the peculiar mode of termination, which, as he stated at the commencement, had not been previously described by any pathologist.

DISEASE OF Mitral VALVES.

Mr. Adams communicated a case of disease of the mitral valves. The subject was a gentleman temperate in his habits, but who had formerly suffered repeated syphilitic attacks, for which he had undergone several mercurial courses. About six years ago he began to feel a difficulty of breathing, and afterwards hæmoptysis and palpitations. Three months ago (on the 3rd of September), while turning in his bed, he perceived a remarkable irregularity in the action of the heart. A few weeks back he came to Dublin, and applied for advice to Mr. Adams, who, on examining him, found that his limbs were anasarcous, and there were evident symptoms of a disease of the heart, but it was doubtful whether it were aneurysm or disease of the valves. The second sound of the heart was accompanied by a sound like that observed in cases of aneurysm. A consultation was then held with Dr. Stokes, who was of opinion that the mitral valves were diseased, and that the death would be very sudden. The treatment adopted consisted in the exhibition of small doses of calomel, squill, and digitalis, together with small bleedings by leeches, which were used in preference to small bleedings from the arm, in consequence of the patient's statement that venesection caused him to faint, and this is objectionable in diseases of the heart. At this time, although the action of the heart was violent, the pulse at the wrist was very weak, the respiration was generally sound, but at night dyspnœa occurred in paroxysms. 'The death was very sudden; it occurred soon after breakfast, while the patient was on the night-chair. The left ventricle was found to be considerably dilated, and the mitral valves indurated, thickened, and infiltrated with a yellowish material, which, in some parts, was ossified. The appearances were well delineated in a drawing produced by Mr. Adams.-Dub. Journ. Med. Sci., March, 1843.

\section{MEDICO-CHIRURGICAL SOCIETY OF} EDINBURGH.

February 1, 1843.

Observations on Impulsive Insanity. By Dr. MACKINNON.

Dr. Mackinnon remarked that insanity was occasionally displayed in an impulse to commit such acts as suicide, homicide, theft, fire-raising, \&c., without any delusion of the understanding, in the common sense of the term, being present. Medical and legal writers, for a long period, exclusively regarded the intellectual faculties, when treating of mental derangement, and omitted all reference to the moral feelings -a part of the mental constitution at least equal in importance to the former. Thus monomania of the intellect was allowed, while monomania of the moral feelings was disregarded. The fact was, however, as remarked by Pritchard, that derangement of the moral feelings was fully more characteristic of insanity than delusion of the intellect. Among the inmates of an asylum, many are always found in whom this moral derangement exists in a greater degree than the intellectual. These prepare us for cases in which the derangement is exclusively in the moral part of the mental constitution. It certainly was sometimes a matter of extreme difficulty to distinguish such cases from others in which criminal acts were committed, for which the perpetrator was justly responsible. The best guides in the diagnosis were the existence or not of hereditary predisposition to mental disease, the presence or absence of the usual motives to crime, the consistency or inconsistency of the acts committed with the previous character of the individual, and, above all, the presence or absence of the physical signs of insanity, such as heat of head, injection of conjunctiva, furred tongue, and derangement of the cuticular and other secretions of the body. Dr. M. proceeded to give examples of impulsive insanity which had come under his observation. In the first case the morale was altogether deranged, and a disposition to commit both homicide and suicide exhibited, while the intellectual faculties, so far from being impaired, were above the average. Hereditary predisposition (a parent committed suicide), and the physical signs of disease were present. In the next case homicidal and suicidal propensities were felt, reasoned on, and deeply regretted by the patient, whose mind was of the highest order. In this case the physical signs of the disease were absent, and would have rendered it a matter of extreme difficulty to have sustained the plea of insanity if homicide had been committed. The whole history, however, conclusively showed a state of mental disease. In the next case the fire-raising and homicidal propensities were displayed. The physical signs were present. In the last case, detailed at length, a propensity to theft, and a wholly perverted morale, were exhibited. Dr. M. alluded to other cases of the same interesting and, in a medico-legal point of view, most important form of insanity.-Edinburgh Monthly Journal.

\section{TREATMENT OF SALIVATION.}

To combat mercurial salivation, I prefer applying acid. hydrochlor. fort. to the gums and tongue when they are ulcerated, repeating the application every day, or every other day. The bleeding of the surface ought not to be an obstacle. The acute pain it produces soon ceases, and nothing equals its beneficial effects. Of course the peculiar indications which may present themselves must not be neglected.M. Ricord on Venereal. 